\title{
Harwardt, Darius (2019): Verehrter Feind. Amerikabilder deutscher Rechtsintellektueller in der Bundesrepublik
}

\author{
Frankfurt/New York: Campus. 560 Seiten. $49 €$
}

\author{
Tobias Adler-Bartels
}

Online publiziert: 26. Oktober 2020

(C) Der/die Autor(en) 2020

Auch wer Hufeisen lediglich auf dem Reiterhof und nicht in den Sozialwissenschaften verortet, wird doch mit Blick auf die linken und rechten Ränder des politischen Spektrums in Deutschland gewisse Berührungspunkte in den teilweise drastischen Verdikten über die Vereinigten Staaten von Amerika erblicken. Sei es als alliierte Sieger- und rücksichtlose Okkupations-, als imperialistische bzw. neoliberale Weltmacht oder als vermeintlich kulturlose Nation von "Yankees“ - antiamerikanische Vorurteile und Stereotype scheinen eine Konstante des radikalen politischen Denkens linker wie rechter Provenienz der Bundesrepublik zu bilden. Der Historiker Darius Harwardt (Universität Duisburg-Essen) hat mit seiner nun publizierten Dissertation „Verehrter Feind. Amerikabilder deutscher Rechtsintellektueller in der Bundesrepublik" eine detaillierte Entwicklungsgeschichte des rechten Antiamerikanismus nach 1945 verfasst und damit gleichzeitig einen wichtigen Beitrag zur ,intellectual history“ der Bundesrepublik geleistet.

In den theoretischen Vorbemerkungen expliziert Harwardt mit Blick auf die akademischen Debatten zunächst aufschlussreich sein Verständnis der forschungsleitenden Begriffe (Neue Rechte, Ideologie, Identität, Intellektuelle). Um den Fallstricken eines methodologischen Zirkelschlusses in Bezug auf den Antiamerikanismus zu entgehen - der vorab Merkmale festlegt, die dann lediglich additiv zugeordnet werden -, zielt Harwardt in seiner Untersuchung auf das offenere Konzept von „Amerikabildern“ (S. 67), welche anhand einer Diskursanalyse einschlägiger rechter Zeitschriften, wie u.a. Nation Europa, Criticón, wir selbst, Deutsche Nationalzeitung, Deutschland-Magazin oder Junge Freiheit, expliziert werden. Indem dabei sogar die Perspektive auf Leserbriefdebatten oder Online-Kommentarspalten geweitet wird, bietet die Monografie tiefe Einblicke in das rechte Milieu und es ist daher für die

T. Adler-Bartels $(\bowtie)$

Institut für Politikwissenschaft, Georg-August-Universität Göttingen, Göttingen, Deutschland

E-Mail: tobias.adler-bartels@uni-goettingen.de 
weitere Forschung umso bedauerlicher, dass sich der Verlag entschieden hat, auf ein Personen- und Sachregister zu verzichten.

Die Arbeit gliedert sich weitgehend chronologisch anhand sogenannter ,kritischer Ereignisse“ (Pierre Bourdieu), die von den rechten Akteurinnen und Akteuren zwingend eine Stellungnahme verlangen. Mit Blick auf die (neu-)rechte Gretchenfrage „Wie hast du es mit Amerika?" blickt Harwardt im Anschluss an eher allgemeinere Überblicke über die Bundesrepublik der Nachkriegszeit und 1968 somit auf die kontroversen Debatten rund um den NATO-Doppelbeschluss, den Zweiten Golfkrieg zu Beginn der 1990er-Jahre sowie die islamistischen Anschläge vom 11.09.2001, wobei jedes Kapitel jeweils durch eine profunde Einführung in den bundesrepublikanischen Kontext eingebettet ist.

Für die revanchistische Rechte in der Frühphase der Bundesrepublik waren die USA als omnipräsente Besatzungs- und Siegermacht ein beliebtes Feindbild, was sich nicht nur an den Verkaufszahlen von Werken wie Ernst von Salomons autobiografischem Roman „Der Fragebogen“ (1951) oder Caspar von Schrenck-Notzings „Charakterwäsche“ (1965) offenbart. Die Polemiken des bekennenden Antiliberalen Armin Mohler über die Vereinigten Staaten erfüllten dabei auch eine legitimierende Funktion, um eine eigenständige, ,neutrale“ Stellung Deutschlands im nunmehr Kalten Krieg zu behaupten. Dass es ausgerechnet dieser ,deutsche Gaullist“" war, der 1967 von amerikanischen Außenpolitikern gebeten wurde, eine Expertise über die europäischen Perspektiven auf Amerika zu verfassen, ist eine interessante neue Begebenheit, die man verblüfft zur Kenntnis nimmt.

Die gesellschaftlichen und politischen Polarisierungen der 1960er- und 1970erJahre sorgten innerhalb des rechten Lagers der deutschen Amerikakritiker und -skeptikerinnen für kontroverse Diskussionen, v. a. mit Blick auf die Beurteilung der Präsidentschaft von Ronald Reagan ab 1981. So wähnten sich Teile der Autorinnen und Autoren von Criticón oder des Deutschland-Magazins als Teil einer deutsch-amerikanischen Allianz im neokonservativen Kampf gegen die Sowjetunion und deren Vasallen. Dagegen regte sich innerhalb des rechten Lagers nicht nur bei bekennenden Neutralisten wie Mohler oder Hans-Dietrich Sander Widerstand - bezog doch die nunmehr entstehende selbsternannte Neue Rechte um einflussreiche Vertreter wie Alain de Benoist oder Henning Eichberg gerade aus ihrem antiimperialistischen Kampf gegen die USA die Legitimation für den sogenannten Befreiungsnationalismus. Der französische Vordenker de Benoist - den Harwardt aufgrund seines immensen Einflusses in den hiesigen rechten Medien für den deutschen Rechtsintellektualismus vereinnahmt - und Eichberg zielten mit ihrer ethnopluralistisch begründeten Kritik auf die vermeintlich universalistischen Ansprüche der so titulierten amerikanischen „One-World“-Ideologie.

Im Wesentlichen kristallisieren sich somit bereits in den Kontroversen der 1970erJahre die Lager heraus, die im weiteren Verlauf die rechten Diskurse maßgeblich prägen: ein radikalisierter Konservatismus, der sich in unmittelbarer Tradition der Weimarer Konservativen Revolution versteht; die Nationalrevolutionären, die Formen und Inhalte der studentischen Protestbewegungen imitierten oder adaptierten und auf Querfrontstrategien setzen; sowie ein sehr heterogenes rechtsextremes Milieu u.a. um die Zeitschrift Nation Europa oder den Grabert-Verlag, in welchem zum Teil unverhohlen der Anschluss an antisemitische und rassistische Positionen 
des Nationalsozialismus gesucht wurde. Dagegen blieben die Versuche, einen deutschen Neokonservatismus nach amerikanischem Vorbild zu etablieren, auf kleine und wenig einflussreiche Kreise am rechten Rand der Unionsparteien beschränkt. Harwardts „Versuch, das Phänomen der ,Neuen Rechten“ zu historisieren“ (S. 37) offenbart somit mit Blick auf die sehr unterschiedlichen Amerikabilder die wesentlichen Inkongruenzen des heterogenen rechten Lagers.

In der Diskussion um die Folgen des Golfkrieges sowie von 9/11 nimmt zunächst die allgemeine politische Debatte einen großen Raum ein. Hier weist Harwardt auch auf die negativen Amerikabilder deutscher Leitmedien (v. a. des Spiegels) hin. Im rechten Milieu wird die These eines nunmehr offenbaren globalen „Kampfes der Kulturen“ (Samuel P. Huntington) breit rezipiert und kontrovers diskutiert. Während Nationalkonservative wie Karlheinz Weißmann sich im Kampf gegen den Islam veranlasst sehen, auf der Seite des ,sanften Hegemon“ (S. 440) jenseits des Atlantik zu stehen, offenbart die von der Jungen Freiheit initiierte Leserdebatte zum Verhältnis zu Amerika weiterhin ein großes Unbehagen. Mit den Gründungen der Blogs „Politically Incorrect“ und „Achse des Guten“ im Jahr 2004 etablieren sich dann neue Formate des Rechtsintellektualismus, die zudem durch ihre proamerikanischen Positionen und libertären Inhalte für eine populistischere Rechte stehen.

Auch wenn die Studie von Harwardt mit ihrer Darstellung des deutschen Rechtsintellektualismus keinen Anspruch auf Vollständigkeit anstrebt, so hätte man sich doch gewünscht, etwas zur Rolle von so einflussreichen Akteuren wie Gerd-Klaus Kaltenbrunner oder Günter Rohrmoser zu erfahren, die trotz ihrer internationalen Perspektiven leider kaum Erwähnung finden. Zudem suggeriert das Werk an vielerlei Stellen, dass die ideelle Entwicklung des deutschen Rechtsintellektualismus nach 1945 lediglich in weiteren Fußnoten zu Carl Schmitt bestünde und zementiert somit einen in rechten Kreisen oftmals gern gepflegten quasi-mythischen Stellenwert des Plettenberger Eremiten. Doch das schmälert nicht den wichtigen Beitrag von Harwardts Werk, welches einen weiten Bogen spannt und gerade durch die klugen Einordnungen auch für Leserinnen und Leser sehr geeignet ist, die sich bisher wenig mit dem Narrensaum des deutschen politischen Denkens auseinandergesetzt haben. Das Werk sei somit als gewinnbringende Lektüre empfohlen, um die gegenwärtigen politischen und gesellschaftlichen Polarisierungen am rechten Rand besser zu verstehen.

Funding Open Access funding enabled and organized by Projekt DEAL.

Open Access Dieser Artikel wird unter der Creative Commons Namensnennung 4.0 International Lizenz veröffentlicht, welche die Nutzung, Vervielfältigung, Bearbeitung, Verbreitung und Wiedergabe in jeglichem Medium und Format erlaubt, sofern Sie den/die ursprünglichen Autor(en) und die Quelle ordnungsgemäß nennen, einen Link zur Creative Commons Lizenz beifügen und angeben, ob Änderungen vorgenommen wurden.

Die in diesem Artikel enthaltenen Bilder und sonstiges Drittmaterial unterliegen ebenfalls der genannten Creative Commons Lizenz, sofern sich aus der Abbildungslegende nichts anderes ergibt. Sofern das betreffende Material nicht unter der genannten Creative Commons Lizenz steht und die betreffende Handlung nicht nach gesetzlichen Vorschriften erlaubt ist, ist für die oben aufgeführten Weiterverwendungen des Materials die Einwilligung des jeweiligen Rechteinhabers einzuholen.

Weitere Details zur Lizenz entnehmen Sie bitte der Lizenzinformation auf http://creativecommons.org/ licenses/by/4.0/deed.de. 